\title{
Parametric recurrence quantification analysis of autoregressive processes for pattern recognition in multichannel electroencephalographic data
}

\author{
Sofiane Ramdani ${ }^{\mathrm{a}, *}$, Anthony Boyer ${ }^{\mathrm{b}}$, Stéphane Caron $^{\mathrm{a}}$, François \\ Bonnetblanc $^{\mathrm{b}, \mathrm{c}}$, Frédéric Bouchara ${ }^{\mathrm{d}}$, Hugues Duffau ${ }^{\mathrm{e}}$, Annick Lesne ${ }^{\mathrm{f}, \mathrm{g}}$ \\ ${ }^{a}$ IDH, LIRMM, CNRS, University of Montpellier, Montpellier, France \\ ${ }^{b}$ CAMIN, INRIA, LIRMM, University of Montpellier, 34090 Montpellier, France \\ ${ }^{c}$ Cognition, Action et Plasticité Sensorimotrice, INSERM U1093, UFR STAPS, \\ Université Bourgogne Franche-Comté, 21078 Dijon, France \\ ${ }^{d}$ Université de Toulon, Aix Marseille University, CNRS, LIS, Toulon, France \\ ${ }^{e}$ Plasticité cérébrale, cellules souches neurales et tumeurs gliales, INSERM U1051, and \\ Institut des Neurosciences de Montpellier, 34295 Montpellier, France \\ ${ }^{f}$ Sorbonne Université, CNRS, Laboratoire de Physique Théorique de la Matière \\ Condensée, LPTMC, F-75252, Paris, France \\ ${ }^{g}$ Institut de Génétique Moléculaire de Montpellier, University of Montpellier, CNRS, \\ Montpellier, France
}

\begin{abstract}
Recurrence quantification analysis (RQA) is an acknowledged method for the characterization of experimental time series. We propose a parametric version of RQA, pRQA, allowing a fast processing of spatial arrays of time series, once each is modeled by an autoregressive stochastic process. This method relies on the analytical derivation of asymptotic expressions for five current RQA measures as a function of the model parameters. By avoiding the construction of the recurrence plot of the time series, pRQA is computationally efficient. As a proof of principle, we apply pRQA to pattern
\end{abstract}

\footnotetext{
${ }^{*}$ Corresponding author

Email address: sofiane.ramdani@umontpellier.fr (Sofiane Ramdani )
} 
recognition in multichannel electroencephalographic (EEG) data from a patient with a brain tumor.

Keywords: Recurrence plots, recurrence quantification analysis, autoregressive stochastic processes, asymptotic recurrence measures, multichannel data, EEG data.

\section{Introduction}

Recurrence plots (RPs) were introduced as a graphical approach for the analysis of chaotic dynamics [1]. A RP is a binary matrix visualizing the pairs $(i, j)$ of states of a dynamical system, visited at times $i$ and $j$, that are closer than a fixed threshold distance $\varepsilon$ in the phase space. Various patterns observed in RPs were associated with specific dynamical properties. For instance, diagonal lines (Fig. 1) are related to the predictability of the dynamics [2, 3, 4]. Accordingly, RPs generated by white noise contain very few diagonals while RPs of deterministic or strongly autocorrelated stochastic signals show more frequent and longer diagonals [5]. The analysis of the vertical lines can reveal dynamical transitions or intermittency [6]. Recurrence quantification analysis (RQA) introduced several quantitative measures based on these patterns [7, 8]. We here explore the application of RQA to multichannel data i.e. a spatially extended array of univariate data, as multichannel electroencephalographic (EEG) data. Our goal is to visualize spatial variations and patterns in a topographic map of local RQA measures. An essential preliminary step, which will be our main focus here, is to develop a computationally efficient RQA for the fast treatment of all the channels.

The development of RQA measures was mainly carried out in the con- 
text of deterministic chaotic dynamics [3, 9, 10]. However, several studies have shown the relevance of RP features also in the pattern recognition field. For instance, Junejo et al. exploited the analogy between RPs and the concept of self-similarity matrices in a descriptor for human action recognition [11. Unthresholded RPs were exploited to predict the dynamics of nonlinear and non-stationary systems through trajectory segmentation [12]. In [13], the frame kernel matrix, which is basically a recurrence matrix involving a Gaussian kernel, was used for temporal clustering of human motion. Faria et al. used RPs to represent time series for plant recognition [14]. The time series classification problem was also addressed using a semi-supervised approach based on a similarity measure derived from cross RQA [15].

Few studies have specifically addressed the statistical properties of RPs generated by random processes. Rohde et al. [16] analyzed statistical features of unthresholded recurrence plots of stationary stochastic processes. Using the Shannon-McMillan-Breiman theorem, Faure \& Lesne [17] related the statistics of RPs of symbolic sequences to the entropy per unit time, with application to Markov chains. Grendár et al. [18] related the asymptotic values of diagonal-based RQA measures to the concept of correlation integral, with application to Markov chains and autoregressive processes.

RPs and RQA have been successful for the analysis of real-world data including noisy biological signals [6, 19, 20], e.g. neurophysiological recordings [21, 22]. Intrinsic noise is included in the dynamics of many models of neurophysiological time series among which the most popular are autoregressive processes of order $p$, denoted $\operatorname{AR}(p)$. Accordingly, we here study the statistical behavior for $\operatorname{AR}(p)$ of five RQA measures obtained from the 
diagonal and vertical features of their infinite-size RPs (with no embedding). The results are based on the improvement of a methodology introduced in our previous studies [23, 24]. By establishing new convergence proofs, we analytically derive refined expressions for the asymptotic values of the RQA measures and their dependence on the $\operatorname{AR}(p)$ parameters. These results offer a novel RQA approach consisting in fitting the experimental signal with an $\operatorname{AR}(p)$ and using the theoretical quantification of its RP, that we name parametric RQA (pRQA). The method is assessed by simulating an AR(6) model fitted on a EEG signal and comparing the RQA measures of the simulated sample paths to their theoretical asymptotic counterparts. We also check the consistency between experimental (from the original EEG signal and the derived RP) and pRQA measures.

The central issue of computational time in the RQA of large real-world data [25, 26, 27] is circumvented in our pRQA method, as it does not require the construction of the RPs. This gain, illustrated on a simulated signal of one million points, makes possible to process multichannel data. We benchmarked pRQA on multichannel EEG recordings with a 64-channel system from a patient with a brain tumor, that can be straightforwardly visualized. Computer memory issues in storing RPs for very long time series [27] are similarly alleviated by pRQA.

\section{Recurrence quantification analysis}

The recurrence plot of a time series comprising $N$ real values $\left(x_{i}\right)_{i=1, \ldots, N}$ is constructed (with no embedding) by computing the binary function

$$
\omega_{i, j}(\varepsilon)=\Theta\left(\varepsilon-\left|x_{i}-x_{j}\right|\right) \quad \text { for }(i, j) \in\{1, \ldots, N\}^{2}
$$


where $\Theta$ is the Heaviside function and $\varepsilon>0$ is some fixed threshold. Thus $\omega_{i, j}(\varepsilon)=1$ for pairs $\left(x_{i}, x_{j}\right)$ closer than the threshold distance $\varepsilon$. The RP is the two-dimensional plot representing as black dots the locations $(i, j)$, called recurrence points, where $\omega_{i, j}(\varepsilon)=1$ (Fig. 1). The points of the main diagonal $(i=j)$ are all recurrence points, and the RP is symmetric with respect to this line [4, 20]. A current extension is to consider RPs similarly constructed from embedded trajectories, where the scalar values $x_{i}$ are replaced with $m$-dimensional time-delayed vectors [28, 29, 30]. Considering RPs without embedding is more relevant for the experimental data and stochastic models investigated below, as it avoids the introduction of two additional parameters (embedding dimension and time delay), and makes analytical computations more tractable (see also [3] for a discussion concerning this issue).

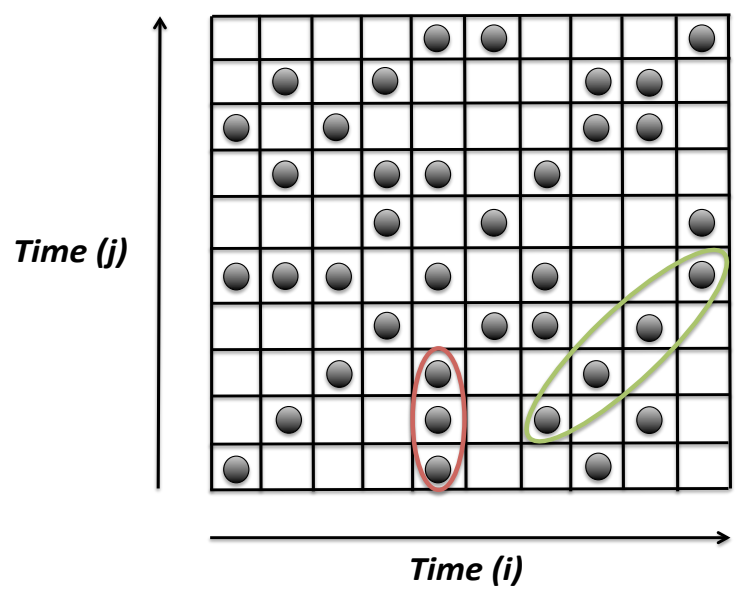

Figure 1: Sketch of a $10 \times 10$ recurrence plot $(\mathrm{RP})$. The black dots are recurrence points, i.e. locations $(i, j)$ where $\left|x_{i}-x_{j}\right| \leqslant \varepsilon$. The green ellipse circles a diagonal line of length 4 , and the red one a vertical line of length 3 .

Recurrence quantification analysis provides a set of measures that can 
be extracted from the RP. The recurrence rate $R E C$ (also denoted $R R$ ) is the fraction of recurrence points in the RP. The percent determinism, DET, is given by the fraction of recurrence points belonging to diagonal lines of length at least $n$ [4, 20]. A third measure is the mean diagonal line length, $L_{d}$. Denoting $D_{k}(\varepsilon)$ the number of diagonal lines of length exactly $k, D E T$ and $L_{d}$ can be respectively expressed as

$$
\operatorname{DET}(\varepsilon, n)=\frac{\sum_{k=n}^{N} k D_{k}(\varepsilon)}{\sum_{k=1}^{N} k D_{k}(\varepsilon)} \text { and } L_{d}(\varepsilon, n)=\frac{\sum_{k=n}^{N} k D_{k}(\varepsilon)}{\sum_{k=n}^{N} D_{k}(\varepsilon)}
$$

For vertical lines, the analog of $D E T$ is called laminarity, $L A M$, while the average vertical line length, $L_{v}$, is called the trapping time (also denoted TT) [4, 6, 20]. Denoting $V_{k}(\varepsilon)$ the number of vertical lines of length exactly $k, L A M$ and $L_{v}$ can be respectively expressed as

$$
\operatorname{LAM}(\varepsilon, n)=\frac{\sum_{k=n}^{N} k V_{k}(\varepsilon)}{\sum_{k=1}^{N} k V_{k}(\varepsilon)} \text { and } L_{v}(\varepsilon, n)=\frac{\sum_{k=n}^{N} k V_{k}(\varepsilon)}{\sum_{k=n}^{N} V_{k}(\varepsilon)}
$$

Due to the symmetry of the RP, the vertical and horizontal lines are equivalent for the latter two measures. The main diagonal is not considered in the computation of these measures. For our results concerning $R E C$, $D E T$ and $L A M$, we will report numerical values between 0 and 1 and not percentages as sometimes found in the literature.

\section{Theoretical context and definitions}

We consider a real-valued, discrete-time, wide-sense stationary, centered Gaussian stochastic process $\boldsymbol{x}$, associated with a sequence of random vari- 
ables $\left(x_{i}\right), i \in \mathbb{N}^{*}$. We denote $\sigma^{2}$ its variance and assume an asymptotically vanishing autocovariance $\gamma$. The asymptotic behavior of $\gamma$ is dominated by an exponential decay for stationary $\operatorname{AR}(p)$ processes. We will use the same symbols for the random variables and their realizations. The infinite-size RP of the process is a random object whose features are characterized by their occurrence probabilities. For a location $(i, j)$ in the RP, a threshold distance $\varepsilon>0$ and a line length $k$, we denote:

- $P_{i, j}(\varepsilon)$, the occurrence probability of a recurrence point in $(i, j)$.

- $P_{i, j}^{k}(\varepsilon)$ and $T_{i, j}^{k}(\varepsilon)$, respectively the probability of occurrence of a diagonal and a vertical line of length $k$, starting from point $(i, j)$.

- $Q_{i, j}^{k}(\varepsilon)$ and $U_{i, j}^{k}(\varepsilon)$, respectively the probability of occurrence of a diagonal and a vertical line of length exactly $k$, starting from point $(i, j)$.

The RP being symmetric, we will only consider the case $i \geqslant j$. These probabilities will be involved below in the definition of asymptotic RQA measures.

Definition 1. For a threshold $\varepsilon>0$ and a line length $n$, the asymptotic RQA measures $R E C^{\infty}(\varepsilon), D E T^{\infty}(\varepsilon, n), L_{d}^{\infty}(\varepsilon, n), L A M^{\infty}(\varepsilon, n)$ and $L_{v}^{\infty}(\varepsilon, n)$ are respectively defined by

$$
\begin{aligned}
& \operatorname{REC}^{\infty}(\varepsilon)=\lim _{(i-j) \rightarrow+\infty} P_{i, j}(\varepsilon) ; \\
& \operatorname{DET}^{\infty}(\varepsilon, n)=\frac{\sum_{k=n}^{+\infty} k Q^{k}(\varepsilon)}{\sum_{k=1}^{+\infty} k Q^{k}(\varepsilon)} ; \\
& L_{d}^{\infty}(\varepsilon, n)=\frac{\sum_{k=n}^{+\infty} k Q^{k}(\varepsilon)}{\sum_{k=n}^{+\infty} Q^{k}(\varepsilon)}, \text { where } Q^{k}(\varepsilon)=\lim _{(i-j) \rightarrow+\infty} Q_{i, j}^{k}(\varepsilon) ;
\end{aligned}
$$




$$
\begin{aligned}
& \operatorname{LAM}^{\infty}(\varepsilon, n)=\frac{\sum_{k=n}^{+\infty} k U^{k}(\varepsilon)}{\sum_{k=1}^{+\infty} k U^{k}(\varepsilon)} ; \\
& L_{v}^{\infty}(\varepsilon, n)=\frac{\sum_{k=n}^{+\infty} k U^{k}(\varepsilon)}{\sum_{k=n}^{+\infty} U^{k}(\varepsilon)}, \text { where } U^{k}(\varepsilon)=\lim _{(i-j) \rightarrow+\infty} U_{i, j}^{k}(\varepsilon) .
\end{aligned}
$$

The next sections present the demonstrations of the convergence of $P_{i, j}, Q_{i, j}^{k}$ and $U_{i, j}^{k}$ when $(i-j) \rightarrow+\infty$. Before, we show that $Q_{i, j}^{k}$ and $U_{i, j}^{k}$ can be expressed using the probabilities $P_{i, j}^{k}$ and $T_{i, j}^{k}$, respectively.

\subsection{Computation of the probability $Q_{i, j}^{k}$ of occurrence of an exact diagonal}

The occurrence of a diagonal of length exactly $k$ starting from point $(i, j)$ implies that the points $(i-1, j-1)$ and $(i+k, j+k)$ are not recurrence

points, whereas no constraint on these points is involved in $P_{i, j}^{k}(\varepsilon)$. With no further assumption on the process $\boldsymbol{x}$, we can write

$$
Q_{i, j}^{k}=\left[P_{i, j}^{k}-P_{i, j}^{k+1}\right]-\left[P_{i-1, j-1}^{k+1}-P_{i-1, j-1}^{k+2}\right]
$$

The term $\left[P_{i, j}^{k}-P_{i, j}^{k+1}\right]$ ensures that the diagonal beyond $(i, j)$ is not longer than $k$. The subtraction of $\left[P_{i-1, j-1}^{k+1}-P_{i-1, j-1}^{k+2}\right]$ ensures that the diagonal starts at $(i, j)$.

\subsection{Computation of the probability $U_{i, j}^{k}$ of occurrence of an exact vertical}

By applying the same reasoning to the probability $U_{i, j}^{k}(\varepsilon)$, we get

$$
U_{i, j}^{k}=\left[T_{i, j}^{k}-T_{i, j}^{k+1}\right]-\left[T_{i, j-1}^{k+1}-T_{i, j-1}^{k+2}\right]
$$

This last result rigorously holds only for vertical or horizontal lines which are not crossing the main diagonal, that is for $(i-j)$ large enough. This is not a limitation as we are interested in the asymptotic case $(i-j) \rightarrow+\infty$. 
Eqs. (4) and (5) ensure that the convergence of the probabilities $Q_{i, j}^{k}$ and $U_{i, j}^{k}$ can be proven from the convergence of $P_{i, j}^{k}$ and $T_{i, j}^{k}$, when $(i-j) \rightarrow+\infty$.

\section{Convergence of the probabilities $P_{i, j}, P_{i, j}^{k}$ and $T_{i, j}^{k}$}

We now prove the convergence of $P_{i, j}, P_{i, j}^{k}$ and $T_{i, j}^{k}$ when $(i-j) \rightarrow+\infty$, that was only numerically assessed in our previous studies [23, 24].

\subsection{Convergence of the probability $P_{i, j}(\varepsilon)$}

Introducing the random variable $y_{i, j}=x_{i}-x_{j}$, the quantity $P_{i, j}(\varepsilon)$ is the probability that $\left|y_{i, j}\right| \leqslant \varepsilon$, it follows that:

Proposition 1. For a given threshold distance $\varepsilon>0$ and $i \neq j$, we have $P_{i, j}(\varepsilon)=\operatorname{erf}\left(\frac{\varepsilon}{\sqrt{2 \alpha_{i, j}}}\right)$, with $\alpha_{i, j}=2\left[\sigma^{2}-\gamma(i-j)\right]$.

Proof. For the non-trivial case $i \neq j, y_{i, j}$ is a centered Gaussian random variable with variance $\alpha_{i, j}=\left\langle x_{i}^{2}\right\rangle-2\left\langle x_{i} x_{j}\right\rangle+\left\langle x_{j}^{2}\right\rangle$, where $\langle$.$\rangle denotes expectation.$ Since the process $\boldsymbol{x}$ is wide-sense stationary, we can write

$$
\alpha_{i, j}=2\left[\sigma^{2}-\gamma(i-j)\right]
$$

Note that $\gamma(-k)=\gamma(k)$. The probability that $\left|y_{i, j}\right| \leqslant \varepsilon$ can be expressed

$$
P_{i, j}(\varepsilon)=\frac{1}{\sqrt{2 \pi \alpha_{i, j}}} \int_{-\varepsilon}^{+\varepsilon} \exp \left(-\frac{y^{2}}{2 \alpha_{i, j}}\right) d y
$$

Using the error function, we finally obtain the expression

$$
P_{i, j}(\varepsilon)=\operatorname{erf}\left(\frac{\varepsilon}{\sqrt{2 \alpha_{i, j}}}\right)
$$

which proves Proposition 1. 
In this latter expression of $P_{i, j}(\varepsilon)$, the quantity $\alpha_{i, j}=2\left[\sigma^{2}-\gamma(i-j)\right]$ is the only term depending on $i$ and $j$. Introducing the integer $q=i-j$ leads to

$$
P_{q}(\varepsilon)=\operatorname{erf}\left(\frac{\varepsilon}{\sqrt{2 \alpha_{q}}}\right)
$$

with $\alpha_{q}=2\left[\sigma^{2}-\gamma(q)\right]$.

Proposition 2. Given a threshold distance $\varepsilon>0, \lim _{q \rightarrow+\infty} P_{q}(\varepsilon)=\operatorname{erf}\left(\frac{\varepsilon}{2 \sigma}\right)$.

Proof. Eq. (9) can be written explicitly

$$
P_{q}(\varepsilon)=\frac{2}{\sqrt{\pi}} \int_{0}^{\frac{\varepsilon}{\sqrt{2 \alpha_{q}}}} e^{-u^{2}} d u
$$

Using an indicator function on the interval $\left[0, \frac{\varepsilon}{\sqrt{2 \alpha_{q}}}\right]$, we have

$$
P_{q}(\varepsilon)=\frac{2}{\sqrt{\pi}} \int_{0}^{+\infty} \mathbf{1}_{\left[0, \frac{\varepsilon}{\sqrt{2 \alpha_{q}}}\right]} e^{-u^{2}} d u
$$

Introducing the function $h_{q}$ defined by $h_{q}(u)=\frac{2}{\sqrt{\pi}} \mathbf{1}_{\left[0, \frac{\varepsilon}{\sqrt{2 \alpha_{q}}}\right]} e^{-u^{2}}$, we obtain

$$
P_{q}(\varepsilon)=\int_{0}^{+\infty} h_{q}(u) d u
$$

Since we consider a stationary process with an asymptotically vanishing autocovariance $\gamma$, we have $\lim _{q \rightarrow+\infty} \gamma(q)=0$, and thus $\lim _{q \rightarrow+\infty} \alpha_{q}=2 \sigma^{2}$. This proves the pointwise convergence of the sequence $\left(h_{q}\right)_{q \in \mathbb{N}^{*}}$ to the function $h$ defined by $h(u)=\frac{2}{\sqrt{\pi}} \mathbf{1}_{\left[0, \frac{\varepsilon}{2 \sigma}\right]} e^{-u^{2}}$, when $q \rightarrow+\infty$. In addition, the inequality $0<h_{q}(u) \leqslant \frac{2}{\sqrt{\pi}} e^{-u^{2}}$ holds for all $q \in \mathbb{N}^{*}$ and $u \in \mathbb{R}^{+}$, which proves that the sequence $\left(h_{q}\right)_{q \in \mathbb{N}^{*}}$ is dominated by an integrable function. Accordingly, Lebesgue's dominated convergence theorem applies and ensures that $\lim _{q \rightarrow+\infty} P_{q}(\varepsilon)=\int_{0}^{+\infty} h(u) d u=\operatorname{erf}\left(\frac{\varepsilon}{2 \sigma}\right)$, which proves Proposition 2. 


\subsection{Convergence of the probability $P_{i, j}^{k}(\varepsilon)$}

Introducing $\mathbf{y}_{i, j}^{k}=\left(x_{i}-x_{j}, x_{i+1}-x_{j+1}, \ldots, x_{i+k-1}-x_{j+k-1}\right)^{T}, P_{i, j}^{k}(\varepsilon)$ is the probability to have $\left\|\mathbf{y}_{i, j}^{k}\right\|_{\infty} \leqslant \varepsilon$, where $\|\cdot\|_{\infty}$ is the maximum norm. As a centered random vector composed of differences of joint normal components of the process $\boldsymbol{x}, \mathbf{y}_{i, j}^{k}$ is Gaussian of dimension $k$ [31, 32]. The components of the (positive definite) covariance matrix $\Omega(i, j)$ of $\mathbf{y}_{i, j}^{k}$ are given by

$$
\Omega_{r, s}(i, j)=\left\langle\left(x_{i+r-1}-x_{j+r-1}\right)\left(x_{i+s-1}-x_{j+s-1}\right)\right\rangle
$$

for $(r, s) \in\{1,2, \ldots, k\}^{2}$. These components can be expressed using the autocovariance $\gamma$ of the original process $\boldsymbol{x}$ as

$$
\Omega_{r, s}(i, j)=2 \gamma(r-s)-\gamma(i-j+r-s)-\gamma(j-i+r-s)
$$

As $\Omega_{r, s}(i, j)$ depends only on the integer $q=i-j$, a convenient notation is

$$
\Omega_{r, s}(q)=2 \gamma(r-s)-\gamma(q+r-s)-\gamma(-q+r-s)
$$

The probability density function (PDF) of $\mathbf{y}_{i, j}^{k}$ is given by the multivariate Gaussian function $f_{q}^{k}$ depending only on the difference $q=i-j$

$$
f_{q}^{k}(\mathbf{y})=\frac{1}{(2 \pi)^{k / 2}|\boldsymbol{\Omega}(q)|^{1 / 2}} \exp \left(-\frac{1}{2} \mathbf{y}^{T} \boldsymbol{\Omega}(q)^{-1} \mathbf{y}\right)
$$

for $\mathbf{y} \in \mathbb{R}^{k}$, and where $|\boldsymbol{\Omega}(q)|$ is the determinant of the matrix $\boldsymbol{\Omega}(q)$. For a fixed $\varepsilon>0$, the probability to have $\left\|\mathbf{y}_{i, j}^{k}\right\|_{\infty} \leqslant \varepsilon$ similarly depends only on $q=i-j$. Denoting $\mathbb{M}(\varepsilon)=\left\{\mathbf{y}: \mathbf{y} \in \mathbb{R}^{k},\|\mathbf{y}\|_{\infty} \leqslant \varepsilon\right\}$, it can be expressed

$$
P_{q}^{k}(\varepsilon)=\int_{\mathbb{M}(\varepsilon)} f_{q}^{k}(\mathbf{y}) d \mathbf{y}
$$

Theorem 1. For a threshold $\varepsilon>0$ and a positive integer $k$, we have $\lim _{q \rightarrow+\infty} P_{q}^{k}(\varepsilon)=P^{k}(\varepsilon)$, with $P^{k}(\varepsilon)=\int_{\mathbb{M}(\varepsilon)} f^{k}(\mathbf{y}) d \mathbf{y}$, where $f^{k}$ is the pointwise limit of the sequence of functions $\left(f_{q}^{k}\right)_{q \in \mathbb{N}^{*}}$, when $q \rightarrow+\infty$. 
Proof. The proof of this result is based on Lebesgue's dominated convergence theorem, as the proof of Proposition 2. See Appendix A for details.

\subsection{Convergence of the probability $T_{i, j}^{k}(\varepsilon)$}

Introducing $\mathbf{z}_{i, j}^{k}=\left(x_{i}-x_{j}, x_{i}-x_{j+1}, \ldots, x_{i}-x_{j+k-1}\right)^{T}, T_{i, j}^{k}(\varepsilon)$ is the probability to have $\left\|\mathbf{z}_{i, j}^{k}\right\|_{\infty} \leqslant \varepsilon$. The components of the covariance matrix $\boldsymbol{\Phi}(i, j)$ of $\mathbf{z}_{i, j}^{k}$, for $(r, s) \in\{1,2, \ldots, k\}^{2}$, are given by

$$
\Phi_{r, s}(i, j)=\left\langle\left(x_{i}-x_{j+r-1}\right)\left(x_{i}-x_{j+s-1}\right)\right\rangle
$$

or in terms of the autocovariance $\gamma$ of the process $\boldsymbol{x}$

$$
\Phi_{r, s}(i, j)=\gamma(0)-\gamma(i-(j+s-1))-\gamma((j+r-1)-i)+\gamma(r-s)
$$

These components depend only on the integer $q=i-j$, according to

$$
\left.\Phi_{r, s}(q)=\gamma(0)-\gamma(q-s+1)\right)-\gamma(-q+r-1)+\gamma(r-s)
$$

Similarly, the PDF of $\mathbf{z}_{i, j}^{k}$ is a multivariate Gaussian function $g_{q}^{k}$

$$
g_{q}^{k}(\mathbf{z})=\frac{1}{(2 \pi)^{k / 2}|\boldsymbol{\Phi}(q)|^{1 / 2}} \exp \left(-\frac{1}{2} \mathbf{z}^{T} \mathbf{\Phi}(q)^{-1} \mathbf{z}\right)
$$

depending only on $q=i-j$. For any fixed $\varepsilon>0$, the probability $T_{i, j}^{k}(\varepsilon)$ to have $\left\|\mathbf{z}_{i, j}^{k}\right\|_{\infty} \leqslant \varepsilon$ can thus be written

$$
T_{q}^{k}(\varepsilon)=\int_{\mathbb{M}(\varepsilon)} g_{q}^{k}(\mathbf{z}) d \mathbf{z}
$$

Theorem 2. For a given threshold $\varepsilon>0$ and a positive integer $k$, we have $\lim _{q \rightarrow+\infty} T_{q}^{k}(\varepsilon)=T^{k}(\varepsilon)$, with $T^{k}(\varepsilon)=\int_{\mathbb{M}(\varepsilon)} g^{k}(\mathbf{z}) d \mathbf{z}$, where $g^{k}$ is the pointwise limit of the sequence of functions $\left(g_{q}^{k}\right)_{q \in \mathbb{N}^{*}}$, when $q \rightarrow+\infty$.

Proof. See Appendix B for the proof. 


\section{Computation of the asymptotic RQA measures}

Using the convergence results of Proposition 2, Theorem 1 and Theorem 2, we now derive explicit expressions of the asymptotic RQA measures defined in Section 3. These five measures $R E C^{\infty}, D E T^{\infty}, L_{d}^{\infty}, L A M^{\infty}$ and $L_{v}^{\infty}$ constitute the basis of parametric RQA (pRQA).

\subsection{Asymptotic recurrence rate $R E C^{\infty}$}

By definition, $R E C^{\infty}(\varepsilon)=\lim _{q \rightarrow+\infty} P_{q}(\varepsilon)$. Proposition 2 leads to

$$
R E C^{\infty}(\varepsilon)=\operatorname{erf}\left(\frac{\varepsilon}{2 \sigma}\right)
$$

\subsection{Asymptotic percent determinism DET ${ }^{\infty}$}

The expression of $D E T^{\infty}(\varepsilon, n)$, Definition 1, involves the limit probability $Q^{k}(\varepsilon)=\lim _{q \rightarrow+\infty} Q_{q}^{k}(\varepsilon)$. From Eq. (4) in Section 3.1 and Theorem 1, it comes

$$
Q^{k}(\varepsilon)=P^{k}(\varepsilon)-2 P^{k+1}(\varepsilon)+P^{k+2}(\varepsilon)
$$

Using this expression, we can rewrite the equation defining $D E T^{\infty}(\varepsilon, n)$

$$
\operatorname{DET}^{\infty}(\varepsilon, n)=\frac{\sum_{k=n}^{+\infty} k\left[P^{k}(\varepsilon)-2 P^{k+1}(\varepsilon)+P^{k+2}(\varepsilon)\right]}{\sum_{k=1}^{+\infty} k\left[P^{k}(\varepsilon)-2 P^{k+1}(\varepsilon)+P^{k+2}(\varepsilon)\right]}
$$

After some algebra, the sums in Eq. 25 can be simplified, leading to

$$
\operatorname{DET}^{\infty}(\varepsilon, n)=\frac{n P^{n}(\varepsilon)-(n-1) P^{n+1}(\varepsilon)}{P^{1}(\varepsilon)}
$$

where $P^{n}(\varepsilon)$ is given in Theorem 1 . 


\subsection{Asymptotic average diagonal line length $L_{d}^{\infty}$}

From Definition 1, the expression of $L_{d}^{\infty}(\varepsilon, n)$ involves the limit probability $Q^{k}(\varepsilon)$. As for $D E T^{\infty}(\varepsilon, n)$, we can rewrite

$$
L_{d}^{\infty}(\varepsilon, n)=\frac{\sum_{k=n}^{+\infty} k\left[P^{k}(\varepsilon)-2 P^{k+1}(\varepsilon)+P^{k+2}(\varepsilon)\right]}{\sum_{k=n}^{+\infty} P^{k}(\varepsilon)-2 P^{k+1}(\varepsilon)+P^{k+2}(\varepsilon)}
$$

After simplification, this expression leads to

$$
L_{d}^{\infty}(\varepsilon, n)=\frac{n P^{n}(\varepsilon)-(n-1) P^{n+1}(\varepsilon)}{P^{n}(\varepsilon)-P^{n+1}(\varepsilon)}
$$

\subsection{Asymptotic laminarity $L A M^{\infty}$}

In the same line, using Definition 1, Eq. (5) (see Section 3.2) and Theorem 2 , the probability $U^{k}(\varepsilon)=\lim _{q \rightarrow+\infty} U_{q}^{k}(\varepsilon)$ can be written

$$
U^{k}(\varepsilon)=T^{k}(\varepsilon)-2 T^{k+1}(\varepsilon)+T^{k+2}(\varepsilon)
$$

Similar computations to those conducted in Section 5.2 yield

$$
L A M^{\infty}(\varepsilon, n)=\frac{n T^{n}(\varepsilon)-(n-1) T^{n+1}(\varepsilon)}{T^{1}(\varepsilon)}
$$

where $T^{n}(\varepsilon)$ is given by Theorem 2 .

\subsection{Asymptotic trapping time $L_{v}^{\infty}$}

According to Definition 1 and using a similar procedure, we get the following expression for the average vertical line length

$$
L_{v}^{\infty}(\varepsilon, n)=\frac{n T^{n}(\varepsilon)-(n-1) T^{n+1}(\varepsilon)}{T^{n}(\varepsilon)-T^{n+1}(\varepsilon)}
$$




\section{Application to EEG data}

Here, we present the application of pRQA to a single EEG signal and multichannel EEG data collected from a right-handed 33-year-old man with a $116.8 \mathrm{~cm}^{3}$ low-grade glioma (grade II) located in the right frontal lobe and extending to anterior temporal regions. These data were collected during a study which complied with the World Medical Association's Code of Ethics (the 1964 Declaration of Helsinki and its amendments).

\subsection{Recording procedure and pre-processing}

Resting state EEG data were collected prior to awake neurosurgery. The patient was asked to rest with eyes closed for at least 1 minute while signals were recorded at 64 scalp sites using a 10-20 system (BioSemi ActiveTwo). Horizontal eye movements were recorded with electrodes placed on the outer left and right canthi. Vertical eye movements were recorded with an electrode placed under the right eye. Two additional electrodes were placed on the left and right mastoids (A1, A2). The BioSemi system's common mode sense electrode served as the reference electrode. Electrophysiological signals were digitized at a $2048 \mathrm{~Hz}$ sampling rate and acquired with ActiView software. Classical EEG pre-processing techniques were then applied using the EEGLAB toolbox [33, 34] for Matlab (The MathWorks, Inc., Natick, MA, USA). The data were re-referenced against the mean of electrodes A1 and A2 and filtered using a highpass FIR filter with a cut-off frequency of $1 \mathrm{~Hz}$ to remove low-frequency drifts. Additional independent component analysis was performed using EEGLAB's binica routine in order to correct for eye-movement artefacts. Each correction underwent visual inspection. The 
resulting 64 signals were down-sampled by a rate of 4 and a 10,000-points time series was selected for each channel. These time series were also standardized i.e. centered and normalized by their standard deviation.

\subsection{Application of $p R Q A$ to a single EEG signal and numerical simulations of the corresponding autoregressive model}

In this section, we compute the asymptotic RQA measures of an $\mathrm{AR}(6)$ model fitting a single-channel EEG corresponding to electrode F2 in the right frontal lobe. We compare these pRQA results to both the standard empirical RQA measures for an ensemble of simulated sample paths generated from the $\mathrm{AR}(6)$ model and the RQA measures obtained directly from the experimental signal, shown in Fig. 2 together with its RP. We underline that AR processes are very efficient and accurate stochastic models for the description and prediction of EEG data [35, 36, 37].

The recorded EEG time series was 10,000-points long and standardized. The order of the AR model was obtained through the estimation of the partial autocorrelation function [38]. The AR modeling was performed using the least-squares approach minimizing the sum of squared forward-prediction errors. The alternative Burg's lattice method [31] was also used, and the corresponding numerical results, not shown here, were almost identical to those obtained through the least-squares approach. We obtained the following model

$$
x_{k}=\sum_{r=1}^{6} a_{r} x_{k-r}+\eta_{k}
$$

with $a_{1}=0.8509, a_{2}=0.0773, a_{3}=0.1089, a_{4}=-0.0487, a_{5}=-0.0459$, $a_{6}=-0.0918$ and a centered white Gaussian noise $\eta$ with variance 0.1781. 


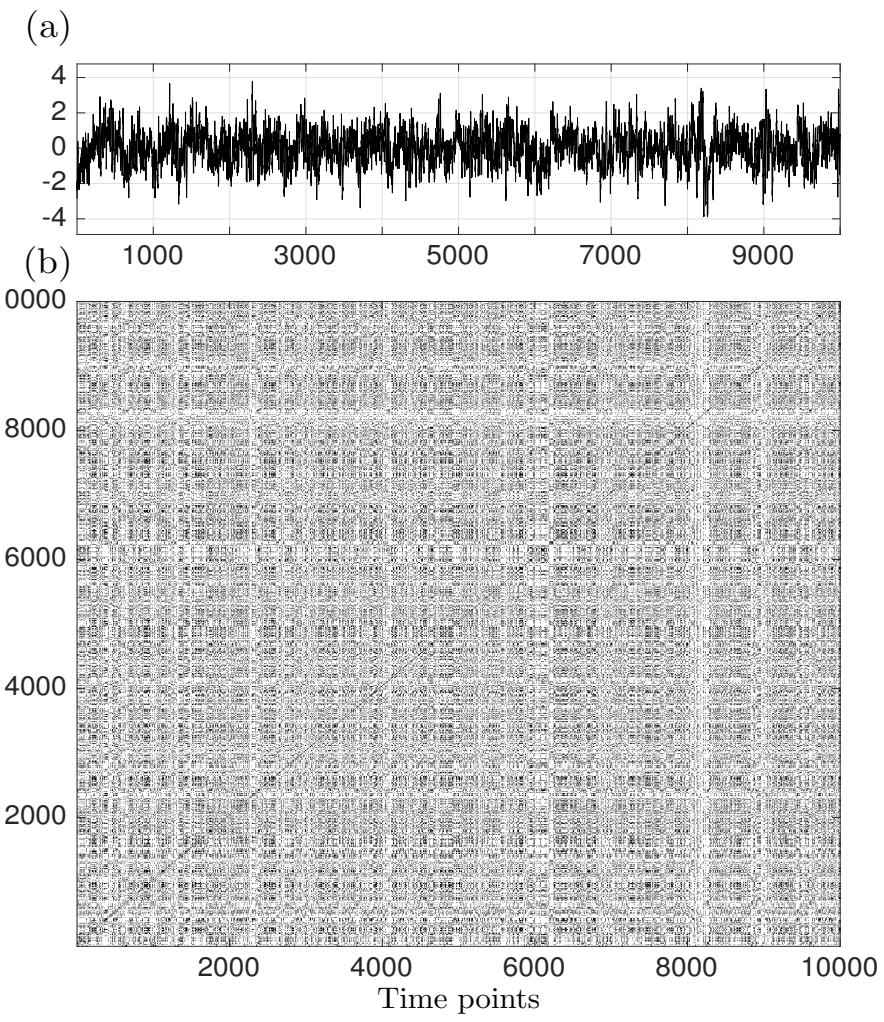

Figure 2: A standardized experimental EEG signal (a) and its RP (b), constructed with $\varepsilon=0.4$ (with no embedding). The corresponding recurrence rate is 0.2247 .

To compute the pRQA measures $D E T^{\infty}(\varepsilon, n), L_{d}^{\infty}(\varepsilon, n), \operatorname{LAM}^{\infty}(\varepsilon, n)$ and $L_{v}^{\infty}(\varepsilon, n)$, we used a threshold $\varepsilon=0.4$ and a minimal line length $n$ ranging from 2 to 10. To compute the probabilities $P^{n}(\varepsilon)$ and $T^{n}(\varepsilon)$ involved in their analytical expressions, we used approximations of multivariate normal probabilities based on the standard algorithm proposed by Genz [39] (see Appendix C). The involved covariance matrices $\boldsymbol{\Omega}$ and $\boldsymbol{\Phi}$ (see Appendix A and B) were obtained from the AR(6) model using the Yule-Walker equations 
[31, 38.

To estimate the corresponding empirical RQA measures, for both the single-channel experimental signal and 30 sample paths 10,000-points long generated by the $\mathrm{AR}(6)$ model, Eq. (32), we used the same threshold $\varepsilon=0.4$. This value was selected to ensure a sufficiently large recurrence rate and a robust estimation of measures based on diagonal and vertical lines for noisy data such as EEG [40, 41]. The values obtained were compared to the pRQA measures for minimal line lengths $n$ ranging from 2 to 10 . For the simulated time series, we computed the mean and standard deviation of the five RQA measures over the 30 realizations of the $\mathrm{AR}(6)$ process. These computations were performed using the Cross Recurrence Plot Toolbox [42] developed by N. Marwan [6].

The value of the asymptotic recurrence rate $R E C^{\infty}$, obtained from Eq. 23) using the standard deviation derived from the AR(6) model was 0.2230. The empirical $R E C$ value for the experimental time series was 0.2247 . For the 30 simulated signals, we obtained a value of $0.2223 \pm 0.0043$ (mean \pm std. dev.). Fig. 3 compares the values of the four other pRQA measures and their counterparts for the simulated and recorded time series, as functions of the minimal line length $n$. For the 30 simulated series, we observe a low variability of the RQA measures around their mean value (plotted in blue). When $n$ varies, the maximal value of the coefficient of variation is $5.91 \%$, $0.43 \%, 10.94 \%$ and $1.62 \%$ for $D E T, L_{d}, L A M$ and $L_{v}$ measures, respectively. These results indicate a very good consistency between the values obtained with pRQA and standard RQA, for both the real signal and the simulation. For smaller values $\varepsilon=0.2$ and $\varepsilon=0.1$, respectively correspond- 
ing to asymptotic $R E C$ values of 0.1126 and 0.0564 , the consistency persists, as expected. Slight deviations are observed between asymptotic and experimental measures for the vertical-based ones $L A M$ and $L_{v}$, for the largest values of $n$ (not shown) due to poorer estimations of standard RQA. Our pRQA method can be seen as an alternative to estimate the measures in such cases, provided that the AR modeling is accurate. We recall that the threshold distance $\varepsilon$ should be adapted to the investigated data and thus chosen to obtain a large enough $R E C$ value.
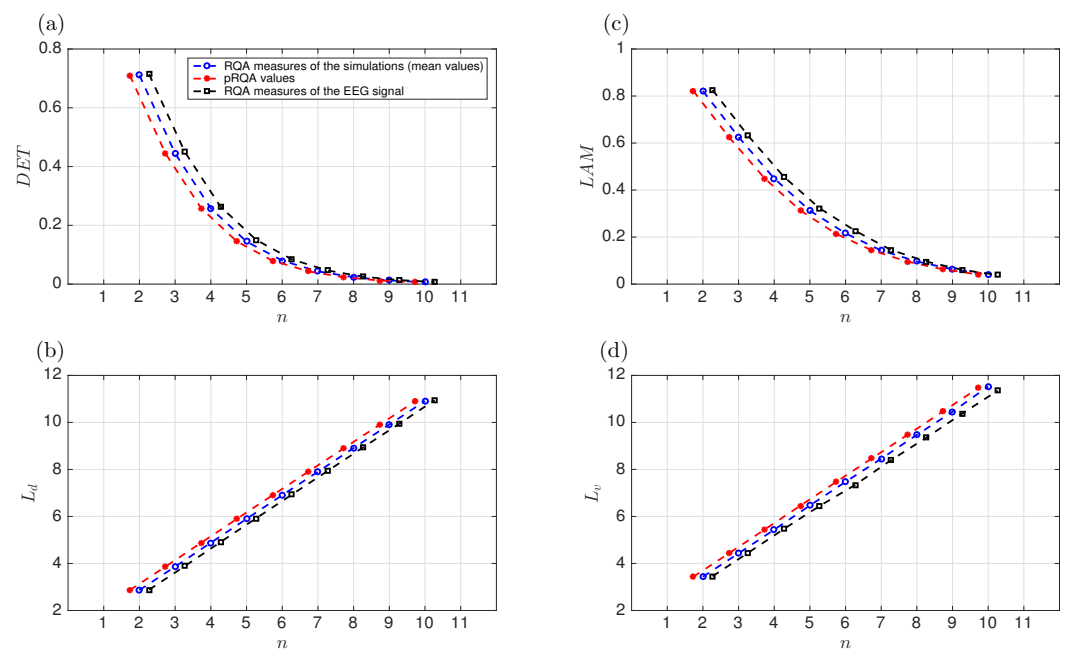

Figure 3: RQA measures (DET (a), $L_{d}(\mathrm{~b}), L A M(\mathrm{c})$ and $\left.L_{v}(\mathrm{~d})\right)$ obtained from a recorded EEG signal with a threshold $\varepsilon=0.4$ and values of the minimal line length $n$ ranging from 2 to 10 . The red points are the pRQA values. The blue ones display the mean of the RQA measures obtained from 30 simulated paths. The black points correspond to the RQA measures of the original EEG signal. For visual clarity, a slight horizontal shift was applied on the red and black points (all having actually integer abscissas). 


\subsection{Application of $p R Q A$ to multichannel EEG data and comparison to stan- dard $R Q A$}

The application of pRQA provided asymptotic RQA measures for each of the 64 EEG sequences. We used the same order $(p=6)$ for their AR modeling, the same threshold $\varepsilon=0.4$ and a minimal line length $n=5$. As a comparison, the standard RQA was applied to the 64 spatially distributed sequences using the same values for $\varepsilon$ and $n$. The selected $\varepsilon$ led to pRQA $R E C$ values with a mean $0.2227 \pm 0.0001$ over the 64 signals and a mean $R E C$ value for their experimental counterparts of $0.2258 \pm 0.0039$. The good correlation between the outcomes of the two approaches is shown by the scatter plots, Fig. 4, of the four RQA measures versus their pRQA counterparts for the 64 EEG signals. The correlation coefficients are 0.9723, 0.9667, 0.9556 and 0.9369 for $D E T, L_{d}, L A M$ and $L_{v}$, respectively. Small systematic biases are globally observed through a slight overestimation of RQA measures values with respect to their pRQA asymptotic counterparts. This could be explained by finite-size effects and artifacts corrupting the signals. We observed that for smaller values $\varepsilon=0.2$ and 0.1 , the good correlations hold, except for the measure $L_{v}$ at large values of $n$ (not shown). Unlike standard RQA, the numerical implementation of pRQA is not sensitive to $\varepsilon$.

Fig. 5 shows the topographic scalp maps obtained from $D E T, L_{d}, L A M$ and $L_{v}$ computed using pRQA or RQA. This figure confirms the good consistency between pRQA and RQA measures. The four measures, for both pRQA and RQA, are maximal in the region of the glioma. Computationally, pRQA outperforms standard RQA, as it does not require the construction of

potentially very large-size RPs. This method thus allows the visualization of 
multichannel EEG data routinely, and possibly in real time.
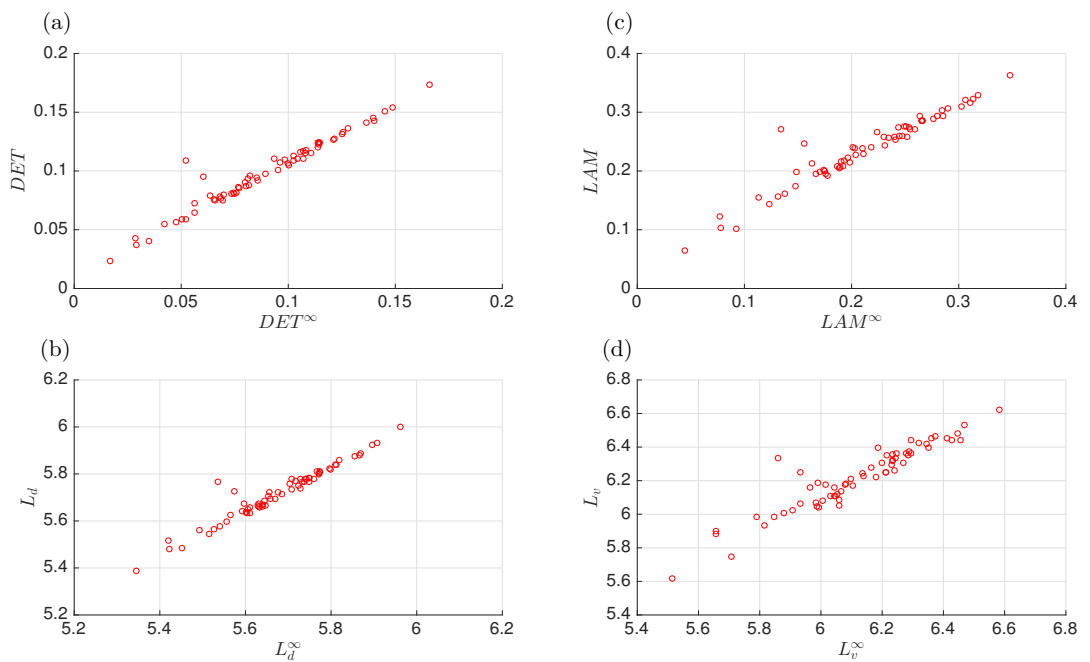

Figure 4: Scatter plot of standard RQA versus pRQA measures DET (a), $L_{d}$ (b), LAM (c) and $L_{v}$ (d) for the spatially distributed 64 EEG signals. A threshold $\varepsilon=0.4$ and a minimal line length $n=5$ were used for the computation of these measures.

The computation time performance of pRQA was quantified by estimating the runtime of processing the 64 EEG signals (each comprising 10,000 values), for minimal line lengths ranging from $n=2$ to 10 , with $\varepsilon=0.4$. The run time included fitting the data with $\operatorname{AR}(6)$ models (for which the computation time was approximately $4 \mathrm{~s}$ ) and calculating the five pRQA measures. We also performed tests with a single simulated signal consisting of one million samples, generated by the $\mathrm{AR}(6)$ model given by Eq. (32). These experiments were conducted with Matlab software through a system consisting of an Intel i7-4770 CPU running at up to $3.4 \mathrm{GHz}$, with 16 Go memory. The runtimes, respectively denoted $t_{1}$ and $t_{2}$, are reported in Table 


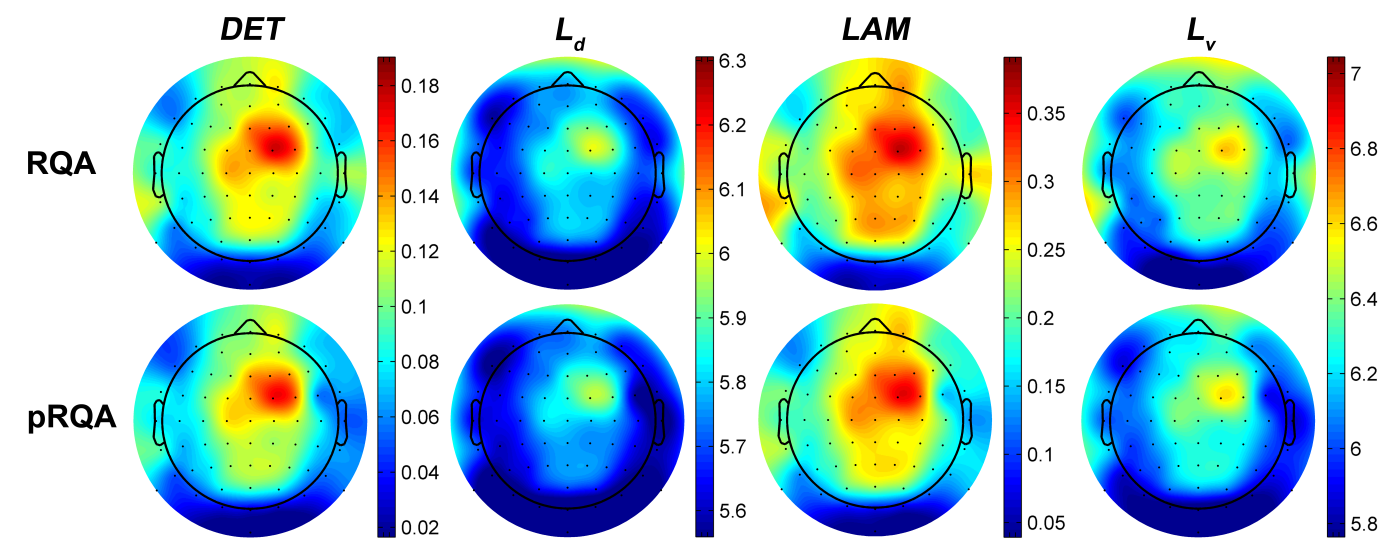

Figure 5: Topographic scalp maps obtained from $D E T, L_{d}, L A M$ and $L_{v}$ measures using standard RQA and pRQA. Same parameters $(\varepsilon=0.4$ and $n=5)$ as for Fig. 4.

1. The processing of 64 EEG sequences of 10,000 points is thus achieved in 9.15 to $34.78 \mathrm{~s}$.

We also performed pRQA for a one million-points simulated signal. The corresponding runtime $t_{2}$ (see Table 1) ranges from $3.95 \mathrm{~s}$ to $4.42 \mathrm{~s}$, from $n=2$ to 10 . A software package (PyRQA), using multiple hardware architectures (GPUs) in a parallel manner, was recently proposed to improve the computational efficiency of RQA of large time series [27]. The authors reported a runtime of $68.94 \mathrm{~s}$ for processing a signal consisting of over one million values, including the computation of 10 RQA measures (with an embedding dimension of 2). A comparison with our approach is not fully relevant because we consider RPs with no embedding and focus on the five most common RQA measures. However, the far shorter runtimes needed in our approach (see Table 1) underline the benefit of avoiding RP construction. 
Table 1: Runtime of pRQA processing for line lengths $n=2$ to 10 applied to 64 EEG signals, each of 10,000-points $\left(t_{1}\right)$ and a single simulated million-points signal $\left(t_{2}\right)$.

\begin{tabular}{lll}
\hline Line length $n$ & $t_{1}(\mathrm{~s})$ & $t_{2}(\mathrm{~s})$ \\
\hline 2 & 9.15 & 3.95 \\
3 & 12.01 & 4.03 \\
4 & 15.30 & 4.04 \\
5 & 18.31 & 4.13 \\
6 & 21.33 & 4.12 \\
7 & 24.77 & 4.23 \\
8 & 28.28 & 4.24 \\
9 & 31.71 & 4.33 \\
10 & 34.78 & 4.42 \\
\hline
\end{tabular}

\section{Discussion}

Our pRQA approach is grounded in a theoretical framework previously introduced [23, 24] for the study of generic stationary Gaussian and fractional Gaussian noise processes. We now proved the convergence of the probabilities $P_{i, j}, Q_{i, j}^{k}$ and $U_{i, j}^{k}$ when $(i-j) \rightarrow+\infty$, which yields the analytical expressions of five asymptotic RQA measures for stationary $\operatorname{AR}(p)$ processes. Despite the fact that $\operatorname{AR}(p)$ processes are fully described by their PDFs and their correlations (or power spectra), our results show that RQA provides a set of measures, with a direct concrete meaning, for discriminating these signals. AR models are not designed to explain nonlinear biological mechanisms but they are very efficient to provide a robust statistical representation of the 
time series. Our work fully fits into this framework: we consider recurrence plots as random objects and we analyze the statistical properties of their patterns. Through the outcomes of pRQA, we are able to quantify some properties of the underlying dynamics such as predictability, which are not explicitly available from the AR model itself.

A technical alternative can be considered in the application to real data. Indeed, the limit probabilities $P^{n}$ and $T^{n}$, respectively given by Theorem 1 and Theorem 2, are related to the autocovariance $\gamma$ of the original process (see Eqs. A.1, A.2 B.1, B.3). The knowledge of this autocovariance is thus sufficient to compute pRQA measures, with no need to fully determine the $\operatorname{AR}(p)$ model. We presented above the results obtained by fitting an $\operatorname{AR}(p)$ model to EEG data and estimating the autocovariance from the model parameters using the Yule-Walker relations. Using the alternative method, we could compute the pRQA measures from the autocovariance directly estimated from the data. The results (not presented here) were almost identical to those depicted in Figs. 4 and 5. The maximal relative errors over the 64 EEG processed signals between the measures obtained through the two approaches were $0.59 \%, 0.05 \%, 0.44 \%$ and $0.08 \%$ for DET, $L_{d}, L A M$ and $L_{v}$, respectively. This can be expected considering the length of the time series (10,000 points) and the limited number of autocovariance values involved in the computation of $P^{n}$ and $T^{n}$. A conceptual advantage of the complete determination of the $\operatorname{AR}(p)$ model is to provide a way to quantify the sensitivity of pRQA results to the model parameters. In addition, the influence of the variance of the error term in the model can be investigated. Our interest in $\operatorname{AR}(p)$ processes was also motivated by the investigation of the statistical 
properties of the RPs of EEG data. However, pRQA approach is not limited to these processes since the conditions for its applicability are the knowledge of the PDF of the process, its stationarity, and an asymptotically vanishing autocovariance. In this case, the probabilities involved in the expressions of pRQA measures can be computed and all theoretical results hold.

The proposed approach also overcomes computer memory issues for the processing of very long time series, when the recurrence matrices cannot be stored in the computing system memory [27]. Consequently, since pRQA does not require the construction of these matrices, it can be very helpful to estimate RQA measures for very large time series all the more since the quality of $\operatorname{AR}(p)$ model estimation increases with the data size.

Another strategy has been proposed to reduce the computation time and memory usage for large data [25, 26]. It involves the numerical approximation of RQA measures based on diagonal or vertical lines (DET, $L_{d}$ and $\left.L A M\right)$ using the concepts of pairwise proximities and stationary states, respectively. In contrast to our methodology, this approach is not probabilistic, hence less appropriate for real-word noisy data best modeled by stochastic processes.

The scatter plots in Fig. 4 confirm the very good correlation between the two sets of measures and also reveal that the standard RQA measures moderately overestimate the corresponding asymptotic values. These deviations could be explained by finite-size effects, artifacts corrupting the signals and the presence of features that cannot be captured by AR models, such as deviations from stationarity and Gaussianity. This issue should be investigated further. Our results indicate that pRQA can be used for investigating multichannel EEG data with recurrence plots measures (see Fig. 5). These 
findings are promising for the investigation of neurophysiological data, for which $\operatorname{AR}(p)$ modeling has been extensively used in the last decades for analyses based on different techniques such as spectral estimation or causality quantifications [35, 36, 37].

Our findings concern RPs constructed without embedding for technical reasons (see Section 2). An extension can be easily envisioned in the case of measures based on diagonals and for RPs constructed with an arbitrary embedding dimension $m$ with a time delay $\tau=1$. Indeed, the statistics of diagonal lines of length $n$ in a RP constructed with an embedding dimension $m$ can be derived from the embedding-free RP diagonal lines of length $(n+$ $m-1)$ [23, 43]. For the general case, further research should be performed.

\section{Conclusion}

We presented pRQA, a parametric RQA version based on modeling experimental data with $\operatorname{AR}(p)$ processes. We analytically derived asymptotic expressions of five main RQA measures, quantitatively characterizing diagonal and vertical structures of the infinite-size RP of these processes. Application to multichannel EEG data demonstrated the relevance of pRQA and its computational efficiency. The extension of our methodology to other RQA measures is possible when the involved probabilities can be computed. This is the case for the Shannon entropy of the frequency distribution of the diagonal line lengths and the $\varepsilon$-entropy of the investigated process as derived from RPs [4, 17, 20, 23]. From an applied point of view, the use of pRQA for pattern visualization in multichannel EEG data should be investigated systematically to confirm on a larger cohort the present proof of principle. The 
pRQA performance in terms of computation time and memory requirement is also promising, with applications to real-time processing and classification of brain activity patterns for brain computer interfaces or emotion recognition [22. Another application could be the characterization of spatio-temporal patterns in electromyographic signals [44].

\section{Acknowledgments}

This research was supported by the LabEx NUMEV project (ANR-10LABX-20) funded by the French government's "Investissements d'Avenir" program managed by the French National Research Agency (ANR), grants from the Institut Universitaire de France, and INSERM laboratory (U1093). S. Ramdani is grateful to V. Kleptsyn and K. Ramdani for useful discussions.

\section{Appendix A. Proof of Theorem 1}

Proof. Proving Theorem 1 is equivalent to demonstrate that

$\lim _{q \rightarrow+\infty} \int_{\mathbb{M}(\varepsilon)} f_{q}^{k}(\mathbf{y}) d \mathbf{y}=\int_{\mathbb{M}(\varepsilon)}\left[\lim _{q \rightarrow+\infty} f_{q}^{k}(\mathbf{y})\right] d \mathbf{y}$

This would follow from Lebesgue's dominated convergence theorem provided that (i) the sequence $\left(f_{q}^{k}\right)_{q \in \mathbb{N}^{*}}$ converges pointwise to a function $f^{k}$; (ii) the functions $f_{q}^{k}$ are dominated by an integrable function $F^{k}$ in the sense that $\forall \mathbf{y} \in \mathbb{M}(\varepsilon),\left|f_{q}^{k}(\mathbf{y})\right| \leqslant F^{k}(\mathbf{y})$ for any positive integer $q$. Eq. 16 shows that the integer $q$ is only involved in the covariance matrix $\Omega(q)$ given by Eq. (15). Given our assumption of a vanishing asymptotic value of the autocovariance function $\gamma$, the limit matrix $\Omega=\lim _{q \rightarrow+\infty} \Omega(q)$ is well-defined and 
its components, for $(r, s) \in\{1,2, \ldots, k\}^{2}$, are given by

$$
\Omega_{r, s}=\lim _{q \rightarrow+\infty} \Omega_{r, s}(q)=2 \gamma(r-s)
$$

This symmetric matrix depends on $k$ through its size only. Eq. (A.1) shows that $\Omega$ is positive definite so that $|\Omega|>0$. The determinant and inverse operations being continuous, the limits of $|\boldsymbol{\Omega}(q)|$ and $\Omega(q)^{-1}$ when $q \rightarrow+\infty$ are given by $|\Omega|$ and $\Omega^{-1}$, respectively. Hence for all $\mathrm{k}, \varepsilon>0$, and $\mathbf{y} \in \mathbb{M}(\varepsilon)$

$$
\lim _{q \rightarrow+\infty} f_{q}^{k}(\mathbf{y})=f^{k}(\mathbf{y})=\frac{1}{(2 \pi)^{k / 2}|\mathbf{\Omega}|^{1 / 2}} \exp \left(-\frac{1}{2} \mathbf{y}^{T} \mathbf{\Omega}^{-1} \mathbf{y}\right)
$$

which proves condition (i). For condition (ii), we analyze the quantity $\mathbf{y}^{T} \boldsymbol{\Omega}(q)^{-1} \mathbf{y}$ for $\mathbf{y} \in \mathbb{M}(\varepsilon)$. The inverse $\boldsymbol{\Omega}(q)^{-1}$ is also positive definite and thus $\mathbf{y}^{T} \boldsymbol{\Omega}(q)^{-1} \mathbf{y}$ defines a positive definite quadratic form. Hence

$$
\mathbf{y}^{T} \boldsymbol{\Omega}(q)^{-1} \mathbf{y} \geqslant \lambda_{q, 1}\|\mathbf{y}\|^{2}
$$

for $\mathbf{y} \in \mathbb{M}(\varepsilon)$, where $\lambda_{q, 1}>0$ is the smallest eigenvalue (among $k$ ) of the matrix $\Omega(q)^{-1}$. Being continuously dependent on the matrix, the eigenvalues of $\Omega(q)^{-1}$ converge to the eigenvalues of the limit matrix $\Omega^{-1}$, when $q \rightarrow+\infty$. Thus, there exists an eigenvalue $\lambda_{1}>0$ of $\Omega^{-1}$ such that $\lim _{q \rightarrow+\infty} \lambda_{q, 1}=\lambda_{1}$. This convergence implies that

$$
\forall \eta>0, \exists q_{1} \in \mathbb{N}^{*}, \forall q \in \mathbb{N}^{*}, q \geqslant q_{1} \Rightarrow\left|\lambda_{q, 1}-\lambda_{1}\right|<\eta
$$

Choosing $\eta=\frac{\lambda_{1}}{2}$, and combining the inequality A.3 with the result A.4, we get for all $q \geqslant q_{1}$ that $\mathbf{y}^{T} \boldsymbol{\Omega}(q)^{-1} \mathbf{y} \geqslant \frac{\lambda_{1}}{2}\|\mathbf{y}\|^{2}$. It follows that, for all $q \geqslant q_{1}$

$$
\exp \left(-\frac{1}{2} \mathbf{y}^{T} \boldsymbol{\Omega}(q)^{-1} \mathbf{y}\right) \leqslant \exp \left(-\frac{\lambda_{1}}{4}\|\mathbf{y}\|^{2}\right)
$$


This result provides an upper bound on the exponential part of the functions $f_{q}^{k}$ (see Eq. (16)). The convergence of the determinant $|\boldsymbol{\Omega}(q)|$ to $|\boldsymbol{\Omega}|$ when $q \rightarrow+\infty$ ensures that there exists a positive integer $q_{2}$ such that $\Rightarrow|\boldsymbol{\Omega}(q)|>$ $\frac{|\Omega|}{2}$ for all $q \geq q_{2}$, and consequently

$$
\frac{1}{(2 \pi)^{k / 2}|\boldsymbol{\Omega}(q)|^{1 / 2}} \leqslant \frac{1}{(2 \pi)^{k / 2}\left(\frac{|\boldsymbol{\Omega}|}{2}\right)^{1 / 2}}
$$

According to Eq. (16) and combining the inequalities (A.5) and (A.6), we conclude that for $q \geqslant \max \left(q_{1}, q_{2}\right)$, we have $0 \leqslant f_{q}^{k}(\mathbf{y}) \leqslant F^{k}(\mathbf{y})$ for all $\mathbf{y} \in$ $\mathbb{M}(\varepsilon)$, where the integrable function $F^{k}$ is defined by

$$
F^{k}(\mathbf{y})=\frac{1}{(2 \pi)^{k / 2}\left(\frac{|\Omega|}{2}\right)^{1 / 2}} \exp \left(-\frac{\lambda_{1}}{4}\|\mathbf{y}\|^{2}\right)
$$

This proves (ii) and completes the proof of Theorem 1.

\section{Appendix B. Proof of Theorem 2}

Proof. The proof, based on Lebesgue's dominated convergence theorem, is very similar to the proof of Theorem 1 . The only difference lies in the expression of the covariance matrix $\boldsymbol{\Phi}(q)$. From Eq. 20) and the asymptotic vanishing of $\gamma$, the limit matrix $\boldsymbol{\Phi}=\lim _{q \rightarrow+\infty} \boldsymbol{\Phi}(q)$ is well-defined with components

$$
\Phi_{r, s}=\lim _{q \rightarrow+\infty} \Phi_{r, s}(q)=\sigma^{2}+\gamma(r-s)
$$

for $(r, s) \in\{1,2, \ldots, k\}^{2}$. As $\boldsymbol{\Omega}$, the matrix $\boldsymbol{\Phi}$ depends on $k$ only through its size $k \times k$. Introducing the notations $\mathbf{J}$ for the $k$-dimensional square matrix with all components equal to 1 and $\boldsymbol{\Gamma}$, whose components are $\gamma(r-s)$, the limit matrix can be written as $\boldsymbol{\Phi}=\sigma^{2} \mathbf{J}+\boldsymbol{\Gamma}$, where all matrices depend on $k$ 
through their size. To transpose the demonstration of Theorem 1, we must prove that the symmetric matrix $\boldsymbol{\Phi}$ is positive definite. Noting that $\mathbf{J}=\mathbf{1 1}^{T}$ where $\mathbf{1}$ is the $k$-dimensional column vector with all components equal to 1 , we have for any real $k$-dimensional column vector $\mathbf{z}$

$$
\mathbf{z}^{T} \boldsymbol{\Phi} \mathbf{z}=\sigma^{2} \mathbf{z}^{T}\left(\mathbf{1 1}^{T}\right) \mathbf{z}+\mathbf{z}^{T} \boldsymbol{\Gamma} \mathbf{z}=\sigma^{2}\left(\mathbf{1}^{T} \mathbf{z}\right)^{T}\left(\mathbf{1}^{T} \mathbf{z}\right)+\mathbf{z}^{T} \boldsymbol{\Gamma} \mathbf{z}
$$

The product $\left(\mathbf{1}^{T} \mathbf{z}\right)$ being a scalar, the quantity $\left(\mathbf{1}^{T} \mathbf{z}\right)^{T}\left(\mathbf{1}^{T} \mathbf{z}\right)$ is a positive number vanishing if $\mathbf{z}=0$. Since $\boldsymbol{\Gamma}$ is positive definite, we conclude that $\boldsymbol{\Phi}$ is also positive definite. This result leads to the expression of the limit probability $T^{k}(\varepsilon)=\lim _{q \rightarrow+\infty} T_{q}^{k}(\varepsilon)$, that is, $T^{k}(\varepsilon)=\int_{\mathbb{M}(\varepsilon)} g^{k}(\mathbf{z}) d \mathbf{z}$, with

$$
g^{k}(\mathbf{z})=\frac{1}{(2 \pi)^{k / 2}|\boldsymbol{\Phi}|^{1 / 2}} \exp \left(-\frac{1}{2} \mathbf{z}^{T} \boldsymbol{\Phi}^{-1} \mathbf{z}\right)
$$

which completes the demonstration of Theorem 2 .

\section{Appendix C. Computation of multivariate normal probabilities}

We briefly present here the method used to numerically compute the multivariate probabilities involved in the expressions of $P^{n}(\varepsilon)$ and $T^{n}(\varepsilon)$ (see Theorems 1 and 2), as no closed-form expression is available for $n \geq 2$. This approach, which was proposed by Genz [39] (see also the appendix of [23]), is based on a stochastic algorithm using a reformulation of the original integral as an integral over the unit hyper-cube after some transformations requiring a Cholesky decomposition of the covariance matrix involved in the multivariate PDF. We illustrate here the method for the case of probability $P^{n}(\varepsilon)$. 
Let $\mathbf{C C}^{T}$ be the Cholesky decomposition of the covariance matrix $\Omega$. If $\left(c_{k, l}\right)$ are the components of matrix $\mathbf{C}$, according to [39], we get

$$
P^{n}(\varepsilon)=\left(v_{1}-u_{1}\right) \int_{0}^{1}\left(v_{2}-u_{2}\right) \cdots \int_{0}^{1}\left(v_{n}-u_{n}\right) \int_{0}^{1} d \mathbf{w}
$$

where $\mathbf{w}=\left(w_{1}, w_{2}, \ldots, w_{n}\right)^{T}, u_{1}=\Psi\left(\frac{a_{1}}{c_{1,1}}\right), v_{1}=\Psi\left(\frac{b_{1}}{c_{1,1}}\right)$, with $\Psi(y)=\frac{1}{\sqrt{2 \pi}} \int_{-\infty}^{y} \exp \left(-\frac{1}{2} \theta^{2}\right) d \theta$, and for $k=2, \ldots, n$

$$
\begin{aligned}
& u_{k}=\Psi\left(\frac{1}{c_{k, k}}\left[a_{k}-\sum_{l=1}^{k-1} c_{k, l} \Psi^{-1}\left(u_{l}+w_{l}\left(v_{l}-u_{l}\right)\right)\right]\right) \\
& v_{k}=\Psi\left(\frac{1}{c_{k, k}}\left[b_{k}-\sum_{l=1}^{k-1} c_{k, l} \Psi^{-1}\left(u_{l}+w_{l}\left(v_{l}-u_{l}\right)\right)\right]\right)
\end{aligned}
$$

A classical Monte-Carlo algorithm is used to compute the integral given by expression (C.1) 39]. For our application, we set $a_{k}=-\varepsilon$ and $b_{k}=\varepsilon$. We used the source code provided by A. Genz.

\section{References}

[1] J. Eckmann, S. Kamphorst, D. Ruelle, Recurrence plots of dynamical systems, Europhys. Lett. 4 (1987) 973-977.

[2] P. Faure, H. Korn, A new method to estimate the Kolmogorov entropy from recurrence plots: its application to neuronal signals, Physica D 122 (1-4) (1998) 265-279.

[3] M. Thiel, M. C. Romano, P. Read, J. Kurths, Estimation of dynamical invariants without embedding by recurrence plots, Chaos 14 (2) (2004) 234-243. 
[4] N. Marwan, M. Romano, M. Thiel, J. Kurths, Recurrence plots for the analysis of complex systems, Phys. Rep. 438 (2007) 237-329.

[5] http://www.recurrence-plot.tk/glance.php.

[6] N. Marwan, N. Wessel, U. Meyerfeldt, A. Schirdewan, J. Kurths, Recurrence plot based measures of complexity and its application to heart rate variability data, Phys. Rev. E 66 (2002) 026702.

[7] J. Zbilut, C. Webber, Embeddings and delays as derived from quantification of recurrence plots, Phys. Lett. A 171 (1992) 199-203.

[8] C. Webber, J. Zbilut, Dynamical assessment of physiological systems and states using recurrence plot strategies, J. Appl. Physiol. 76 (1994) 965-973.

[9] G. Robinson, M. Thiel, Recurrences determine the dynamics, Chaos 19 (2) (2009) 023104.

[10] Y. Hirata, K. Aihara, Devaney's chaos on recurrence plots, Phys. Rev. E 82 (3) (2010) 036209.

[11] I. N. Junejo, E. Dexter, I. Laptev, P. Perez, View-independent action recognition from temporal self-similarities, IEEE Trans. Pattern Anal. Mach. Intell. 33 (2011) 172-185.

[12] H. Yang, T. Bukkapatnam, Satish, L. G. Barajas, Local recurrence based performance prediction and prognostics in the nonlinear and nonstationary systems, Pattern Recognition 44 (2011) 1834-1840. 
[13] F. Zhou, F. De La Torre, J. K. Hodgins, Hierarchical aligned cluster analysis for temporal clustering of human motion, IEEE Trans. Pattern Anal. Mach. Intell. 35 (2013) 582-596.

[14] F. A. Faria, J. Almeida, B. Alberton, L. P. C. Morellato, R. da S. Torres, Fusion of time series representations for plant recognition in phenology studies, Pattern Recogn. Lett. 83 (2016) 205-214.

[15] L. de Carvalho Pagliosa, R. Fernandes de Mello, Semi-supervised time series classification on positive and unlabeled problems using crossrecurrence quantification analysis, Pattern Recognition 80 (2018) 53-63.

[16] G. K. Rohde, J. M. Nichols, B. M. Dissinger, F. Bucholtz, Stochastic analysis of recurrence plots with applications to the detection of deterministic signals, Physica D 237 (5) (2008) 619-629.

[17] P. Faure, A. Lesne, Recurrence plots for symbolic sequences, Int. J. Bifurcat. Chaos 20 (06) (2010) 1731-1749.

[18] M. Grendár, J. Majerová, V. Špitalský, Strong laws for recurrence quantification analysis, Int. J. Bifurcat. Chaos 23 (2013) 1350147.

[19] S. Ramdani, G. Tallon, P. L. Bernard, H. Blain, Recurrence quantification analysis of human postural fluctuations in older fallers and nonfallers, Ann. Biomed. Eng. 41 (8) (2013) 1713-1725.

[20] C. Webber, N. Marwan, Recurrence Quantification Analysis, Springer International Publishing, 2015. 
[21] N. Marwan, A. Meinke, Extended recurrence plot analysis and its application to ERP data, Int. J. Bifurcat. Chaos 14 (02) (2004) 761-771.

[22] Y.-X. Yang, Z.-K. Gao, X.-M. Wang, Y.-L. Li, J.-W. Han, N. Marwan, J. Kurths, A recurrence quantification analysis-based channel-frequency convolutional neural network for emotion recognition from EEG, Chaos 28 (8) (2018) 085724.

[23] S. Ramdani, F. Bouchara, J. Lagarde, A. Lesne, Recurrence plots of discrete-time Gaussian stochastic processes, Physica D 330 (2016) 1731.

[24] S. Ramdani, F. Bouchara, A. Lesne, Probabilistic analysis of recurrence plots generated by fractional Gaussian noise, Chaos 28 (8) (2018) 085721.

[25] D. Schultz, S. Spiegel, N. Marwan, S. Albayrak, Approximation of diagonal line based measures in recurrence quantification analysis, Phys. Lett. A 379 (2015) 997-1011.

[26] S. Spiegel, D. Schultz, N. Marwan, Approximate recurrence quantification analysis (aRQA) in code of best practice, in: C. Webber, C. Ioana, N. Marwan (Eds.), Recurrence Plots and Their Quantifications: Expanding Horizons. Springer Proceedings in Physics, Vol. 180, Springer, Cham, 2016, pp. 113-136.

[27] T. Rawald, M. Sips, N. Marwan, PyRQA - conducting recurrence quantification analysis on very long time series efficiently, Comput. \& Geosci. 104 (2017) 101-108. 
[28] N. Packard, J. Crutchfield, J. Farmer, R. Shaw, Geometry from a time series, Phys. Rev. Lett. 45 (1980) 712-716.

[29] F. Takens, Detecting strange attractors in turbulence, in: Dynamical systems and turbulence, Warwick 1980, Springer, 1981, pp. 366-381.

[30] H. Kantz, T. Schreiber, Nonlinear time series analysis, Cambridge University Press, 2nd ed., 2004.

[31] A. Papoulis, S. Pillai, Probability, random variables and stochastic processes, 4th Edition, McGraw-Hill Higher Education, 2002.

[32] C. Rasmussen, C. Williams, Gaussian processes for machine learning, MIT Press, 2006.

[33] A. Delorme, S. Makeig, EEGLAB: an open source toolbox for analysis of single-trial EEG dynamics including independent component analysis, J. Neurosci. Methods 134 (1) (2004) 9-21.

[34] https://sccn.ucsd.edu/wiki/EEGLAB.

[35] W. Gersch, Spectral analysis of EEG's by autoregressive decomposition of time series, Math. Biosci. 7 (1-2) (1970) 205-222.

[36] J. Pardey, S. Roberts, L. Tarassenko, A review of parametric modelling techniques for EEG analysis, Med. Eng. \& Phys. 18 (1) (1996) 2-11.

[37] E. Pereda, R. Q. Quiroga, J. Bhattacharya, Nonlinear multivariate analysis of neurophysiological signals, Prog. Neurobiol. 77 (1-2) (2005) 1-37. 
[38] G. E. Box, G. M. Jenkins, G. C. Reinsel, G. M. Ljung, Time series analysis: forecasting and control, John Wiley \& Sons, 2015.

[39] A. Genz, Numerical computation of multivariate normal probabilities, J. Comput. Graph. Stat. 1 (1992) 141-149.

[40] M. Thiel, M. C. Romano, J. Kurths, R. Meucci, E. Allaria, F. T. Arecchi, Influence of observational noise on the recurrence quantification analysis, Physica D 171 (3) (2002) 138-152.

[41] N. Marwan, How to avoid potential pitfalls in recurrence plot based data analysis, Int. J. Bifurcat. Chaos 21 (04) (2011) 1003-1017.

[42] http://tocsy.pik-potsdam.de/CRPtoolbox.

[43] T. March, S. Chapman, R. Dendy, Recurrence plot statistics and the effect of embedding, Physica D 200 (1-2) (2005) 171-184.

[44] D. Farina, L. Fattorini, F. Felici, G. Filligoi, Nonlinear surface emg analysis to detect changes of motor unit conduction velocity and synchronization, J. Appl. Physiol. 93 (2002) 1753-1763. 